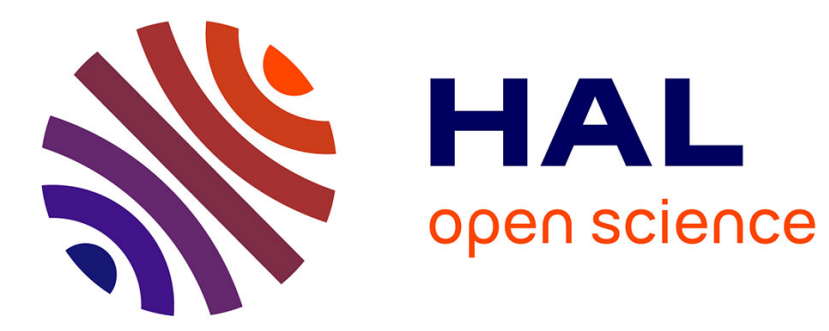

\title{
Integrated strategic and tactical optimization of animal-waste sourced biopower supply chains
}

Kunal Kumar, Christian Javier Clavijo Lopez, Oscar Augusto Tellez Sanchez, Amit Guptá, Olivier Péton, Thomas G. Yeung, Adrien Vanuxem

\section{- To cite this version:}

Kunal Kumar, Christian Javier Clavijo Lopez, Oscar Augusto Tellez Sanchez, Amit Guptá, Olivier Péton, et al.. Integrated strategic and tactical optimization of animal-waste sourced biopower supply chains. IESM 2015, University of Seville, University of Valenciennes, Oct 2015, Sevilla, Spain. hal01232434

\section{HAL Id: hal-01232434 \\ https://hal.science/hal-01232434}

Submitted on 23 Nov 2015

HAL is a multi-disciplinary open access archive for the deposit and dissemination of scientific research documents, whether they are published or not. The documents may come from teaching and research institutions in France or abroad, or from public or private research centers.
L'archive ouverte pluridisciplinaire HAL, est destinée au dépôt et à la diffusion de documents scientifiques de niveau recherche, publiés ou non, émanant des établissements d'enseignement et de recherche français ou étrangers, des laboratoires publics ou privés. 


\title{
Integrated strategic and tactical optimization of animal-waste sourced biopower supply chains
}

\author{
(presented at the $6^{\text {th }}$ IESM Conference, October 2015, Seville, Spain) (c) $\mathrm{I}^{4} \mathrm{e}^{2} 2015$
}

\author{
Kunal Kumar, \\ Christian Javier Clavijo Lopez \\ Oscar Augusto Tellez Sanchez, Amit Gupta \\ École des Mines de Nantes, \\ Nantes, France \\ $\{k k u m a r 13$, cclavi13, otello13, agupta13\} \\ @etudiant.mines-nantes.fr
}

\author{
Olivier Péton, Thomas G. Yeung \\ École des Mines de Nantes \\ Institut de Recherche en Communications et \\ Cybernétique de Nantes (IRCCyN, UMR CNRS 6597) vanuxem@sol3d.com \\ Nantes, France \\ \{olivier.peton, thomas.yeung \\ @ mines-nantes.fr
}

\begin{abstract}
Many models have been recently developed for the optimization of biomass related supply chains. However, models for biopower supply chains powered by animal waste have not received much attention yet. In this paper, we propose a mixed integer linear programming model for supplier selection and procurement planning for a biopower plant. The model integrates time window constraints for the collection of animal waste as well as inventory constraints. We show that the model is intractable with a state-of-the art commercial solver and propose a heuristic approach based on the Adaptive Large Neighbourhood Search (ALNS) framework. We show the efficiency of this approach on a case study in central France.
\end{abstract}

\section{INTRODUCTION}

The declining reserves of fossil fuels and an ever-increasing demand for energy has forced many economies to gradually shift their dependency for energy derived from non-renewable to renewable sources. The shift can be seen from the fact that between 2003 and 2012, the gross energy consumption of energy derived from renewable sources in France has increased from $12.1 \%$ to $15.6 \%$ [1].

Among the various sources of renewable energy produced in France, biomass holds a major share with 52\% [2]. Owing to a large agricultural area and immense resources of animal waste, the production of energy from biomass holds a lot of potential in France [3]. Moreover, the potential of biomass to produce energy in different forms - as biofuel (bioethanol) and, biopower (electricity), is really beneficial as well. At present, there exist more than 230 active biomass processing plants worth an installed capacity of $110 \mathrm{MWe}$ and these figures are expected to triple by the year 2020 [4]. This boost in the number of biomass processing plants is due to the increased feed-in tariffs and subsidies provided to the producers.

However, despite the abundance and ease of availability of biomass (agricultural and animal waste), producing energy from biomass is costlier than other sources [5]. In particular, transportation cost of animal waste is an issue because of its high water content [6].

Thus, it is imperative to produce energy at the lowest possible costs for biomass to remain an attractive source of energy in the future.
Biomass collected from agricultural farms can be broadly divided into two categories waste coming from plants (agricultural biomass) and animal waste. Agricultural waste is generally produced during the harvesting season in high quantities. While on the other hand, animal waste is characterized by irregular production, and in small quantities. This imposes time-windows on the collection of animal waste, and thus differentiates it with the agricultural waste. In this paper, we consider the strategic selection of animal waste suppliers for a biopower plant and the tactical optimization of the collection schedule at suppliers. In must be noted here that the biomass considered in this study is restricted to animal waste, due to its characteristics which make it different from other biomass supply chains. In section II, we review the related literature to better position our work in the corresponding field of research. In section III, we propose a mixed integer linear programming (MILP) formulation. The MILP model minimizes the total costs, by taking into account the transportation costs incurred while procuring the biomass. We take into account the timewindows (minimum and maximum gap between consecutive deliveries) to align this problem closer to practice. The model also takes into account several practical constraints such as the collection of a minimum percentage of biomass from a selected supplier. This constraint does not exist in all situations, but is still common in many real-life situations. In section IV, we present a case study in central France and show that the CPLEX solver cannot solve it within acceptable computation time. In section V, we propose an Adaptive Large Neighborhood Search (ALNS) [7], [8] meta-heuristic for the problem. We also run scenarios with different plant capacities and time discretizations. Section VI concludes this paper, and maps issues for further research.

\section{LiterATURE SURVEY}

Extensive literature reviews on the biomass supply chains have been proposed by [9], [10] and [11] and can be referred for the current state of the art of the problem. In their literature review, Eskandarpour et al. [12] identify biomass to bioenergy supply chains as the leading application area of sustainable supply chain network design.

Studies have been made to reduce costs by the optimization of the biomass to bioethanol supply chains which 
take into account the various aspects of the production from harvesting, transportation, and handling from the supply to the end-customer (see [13], [14], [15], [16] or [17]). However, these papers mainly focus on bioethanol production, which have different characteristics compared to biopower production systems. In bioethanol production systems, the end-product is usually supplied to several customers and the production is guided through the stochastic demand for the product. However, in case of biopower production systems, the output is injected into the power grids which serve the local populations. It is thus independent of the customers demand and boundedabove by the treatment capacity of the facility at each period.

Further, there have been studies which take into consideration the different types of biomass like switch-grass [17], cotton-stalk [18] and miscanthus [19] for the production of energy products. Most of these types of biomass are agricultural products and offer high seasonality due to limited periods of harvesting. Consequently, long-term storage is provided as a solution during seasons of no production [20]. As per the knowledge of the authors, there are no studies which consider animal waste as the input for biopower plants.

The procurement of biomass deals with two sub-problems (a) selection of sites from where the biomass waste can be collected and, (b) determination of the procurement plan from each selected site such that the biomass equivalent to the processing capacity of the plant is collected. While the first decision is made for a few years (strategic), the latter decision is taken for shorter periods, usually weeks or months (tactical). The selection of farms would affect the subsequent procurement schedule, which may be globally sub-optimal. Thus, it would be only beneficial to solve the two sub-problems as an integrated global problem, as is done by [16].

The supply chain design for biomass processing industries has not received much attention in the research community until recently. However, recently this topic has interested a lot of researchers due to the growing awareness towards efficient energy production from renewable sources. Several optimization models have been proposed for decisions at strategic, tactical and operational levels. At the strategic level, the main problems are facility location and capacity determination. These problems have been studied by [16], [21] and [22] among others. All of these studies propose MILP models to solve these problems. However, most of these problems are focused on biomass to bioethanol supply chains. At the tactical level, the key decisions include procurement schedules, transportation fleet management and inventory monitoring. These problems have been studied mainly for bioethanol production by several types of biomass. The studies have been made on miscanthus [19], wood residues [23] and cotton stock [18]. The studies have been missing on supply chains with animal waste which are very different at operation and tactical levels.

There have been a few studies which have tried to integrate the two levels of decision as a single problem. Several mathematical models have been proposed to find the optimal facility location and biomass collection schedules [15], [17]. In addition to the above problem, a MILP model to address the problem of farm selection, resource allocation and operating schedule has been proposed in a study by Lin et al. [16]. This is one of the most comprehensive models for biomass to bioethanol supply chains. However, this study is focused on agricultural waste and does not take into the time-windows imposed by animal waste.

Figueirido et. al. [24] presents a systematic approach for the design of biopower supply chains with animal waste as biomass. However, the study does not present any optimization models for the design of the supply chains. Moreover, it proposes the selection of farms through a qualitative method and does not consider the time-windows.

As far as solution methods are concerned, most biomass supply chain design problems are solved with MILP and MINLP solvers (see for example [15]- [17]) which sometimes require extremely long computation times. With the addition of time-windows the problem becomes even harder, especially for a large number of farms and finer time discretization. Thus, we need a method which can provide good results in reasonable amount of time. As far as heuristic and metaheuristic methods are concerned, no method seems to be widely used by different authors. Adaptive Large Neighbourhood Search (ALNS) has proved to be really efficient for solving optimization problems such as vehicle routing problems, arc scheduling problems, pollution routing, snow-plowing and timetabling problem (see [8], [25], [26], [27], [28], [29], [30] or [31]). However, for solving supply chain network design (SCND) problems, the use of Large Neighbourhood Search (LNS) has been explored by a few studies (see ref. [32] or [33]), but to the best of our knowledge, LNS (or ALNS) has not been used yet to solve integrated strategic and tactical problems, especially for biomass supply chain design problems.

The literature survey clearly identifies the need for studying biomass processing supply chains for animal waste which take into account its special characteristics. In addition to that, we need a solution method which provides good results in reasonable amount of time. Thus, our study tries to fill in these gaps by providing a model for the selection of farms and the determination of procurement schedules which take into account the inherent time-windows.

\section{PROBLEM DESCRIPTION}

In this problem, we try to optimize the procurement plan for a single biomass processing facility, with known monthly maximal demand, at integrated strategic and tactical level. It offers several characteristics like seasonal supply, timewindows on collection trips which make the problem interesting. We only consider the upstream leg of the supply chain as the output from the plant is injected into the grid. Downstream actors are not considered as the power is injected directly into the electricity grids. We also consider the facility as a black box, and any activity within the plant is out of the scope of this model.

We consider a single biomass processing facility for a given maximal capacity equivalent to the energy output of $C$ tons of biomass per day. The production is a continuous process and exactly $C$ tons of biomass (animal waste, in this case) must be provided each day. The biomass can be procured from a set $F$ of candidate farms, which can be selected to be long-term suppliers of biomass. The decision of selection or non-selection remains unchanged for the whole planning horizon $T$. Each farm $i \in F$ is located at a distance di from the facility. The availability of biomass at each farm depends 
on the farm size and shows high seasonality. For each period $t \in T$, biomass availability at farm $i$ is given as $P_{i t}$. The length of each period $t \in T$ is $n$ days.

Animal waste offers specific characteristics compared to the agricultural waste. Although it is not limited to small periods (e.g. harvesting), it still offers a seasonal trend. Moreover, there are conditions imposed on the collection of animal waste due to practical reasons. The production of animal waste is spread both spatially and temporally; therefore, all the animal waste is usually assembled together after a certain period of time which could be of the order of a few weeks. This fact introduces a minimum gap between consecutive collection trips, as before this period the animal waste may not be accumulated. Two consecutive collections at a farm must be separated by at least $g_{\min }$ periods.

Moreover, animal waste usually has a higher rate of biodegradation than the agricultural waste, due to its higher moisture content. Its semi-liquid state renders it difficult to store and handle. Thus, long-term storage is avoided [34], [35]. This imposes a restriction on the maximum gap of $g_{\max }$ periods between two consecutive collections at a farm.

We assume that all the manure collected beyond the gap of $g_{\max }$ periods is rendered invaluable due to excessive moisture content and therefore, low heating value. Additionally in some cases, mutual agreement dictates a collection of at least $\alpha \%$ of the total biomass available at any farm, in case a farm is selected as a supplier. However, to maintain generality, this constraint can be easily relaxed by setting the value of $\alpha$ to zero in cases where this condition does not hold true. The quantity Wit of biomass left in the farm after each collection is disposed off and is not available anymore in subsequent periods. If there is no collection at farm $i$ during period $t-1$, then the quantity $J_{i t}$ of biomass available at period $t$ is calculated as $J_{i t}=$ $J_{i, t-1}+P_{i t}$.

In case the total available biomass from the selected farms is less than the required capacity of $n C$ for the period, a quantity $E_{t}$ of biomass is secured from non-contractual suppliers (not selected) at an additional cost or penalty of A/ton.

Furthermore, storage of collected biomass at the facility is of importance as well. Biomass is degradable and loses its energy value over time due to absorption of moisture. Thus, it is really important to process the biomass before its energy content reaches a threshold below which it is deemed as unacceptable. Any consumption until $K$ days from the day of collection is considered acceptable. Any processing beyond that would result in a reduced production of energy, and hence a loss of $L E U R /$ ton. This cost is in addition to the standard inventory costs for the biomass within the plant, charged at $H E U R /$ ton/period.

The optimization problem consists of selecting a set of farms and planning the procurement at each farm in the time horizon while minimizing the total costs. The various parameters involved in the problem are defined in Table I. The variables of the mathematical model are introduced in Table II.

For the given notations, the problem can be modeled by the MILP (1)-(13).
TABLE I.

PARAMETERS USED IN THE MATHEMATICAL MODEL.

\begin{aligned} & \hline$F$ Pet of farms \\ &$T$ Pet of time periods in the planning horizon \\ &$n$ Length of each time period (in weeks) \\ &$C$ Processing capacity of the facility per day (in tons) \\ &$K$ Number of periods before biomass start losing its power (in weeks) \\ &$P_{i t}$ The biomass availability at farm $i \in F$ during period $t \in T \\ & d_{i}$ Distance of farm $i \in F$ from biopower plant (in km) \\ &$g_{\min }$ Maximum time gap between two consecutive collections for each farm \\ & (in weeks) \\ &$g_{\max }$ Minimum time gap between two consecutive collections for each farm \\ & (in weeks) \\ &$T_{c}$ Inventory cost in the biopower plant (in /ton/week) \\ &$A$ Cost of transport per ton-distance biomass collected (/km-ton) \\ &$L$ Cost (loss of value + handling + storage) incurred to store biomass for \\ & more than $K$ days (in /ton/week) \\ & \hline\end{aligned}

TABLE II. PARAMETERS USED IN THE MATHEMATICAL MODEL.

\begin{tabular}{|c|c|}
\hline \multicolumn{2}{|r|}{ Continuous material flow variables (in tons) } \\
\hline$I_{t}$ & Total inventory of biomass at the biopower plant during period $t \in T$ \\
\hline$J_{i t}$ & Total availability of biomass at farm $i \in F$ during period $t \in T$ \\
\hline$I_{t}^{\prime}$ & $\begin{array}{l}\text { Total amount of biomass that stays for more than } K \text { days at the } \\
\text { facility during period } t \in T\end{array}$ \\
\hline$E_{t}$ & Quantity of additional biomass bought during period $t \in T$ \\
\hline$W_{i t}$ & Amount of biomass discarded after each collection \\
\hline \multicolumn{2}{|r|}{ Binary and Integer Decision Variables } \\
\hline$Y_{i}$ & 1 , if a farm $i \in F$ is selected; 0 , otherwise \\
\hline$X_{i t}$ & 1 , if a collection is made from a farm $i \in F$ during period $t \in T$ \\
\hline$Q_{i t}$ & $\begin{array}{l}\text { quantity of biomass collected from a farm } i \in F \text { during period } t \in T \\
\text { (in tons) }\end{array}$ \\
\hline
\end{tabular}

$\max \quad T_{c} \sum_{t \in T} \sum_{i \in F} Q_{i t} d_{i}+\sum t \in T\left(H I_{t}+A E_{t}+L I_{t}^{\prime}\right)$

s.t.

$$
I_{t}=I_{t-1}+\sum_{i \in F} Q_{i t}+E_{t}-n C \quad \forall t \in T-\{0\}
$$

$$
I_{t} \leq K C+I_{t}^{\prime} \quad \forall t \in T
$$

$J_{i,(t-1)}+P_{i t}=Q_{i t}+J_{i t}+W_{i t} \quad \forall i \in F, t \in T-\{0\}$

$$
W_{i t} \leq M_{i} X_{i t} \quad \forall i \in F, t \in T
$$

$$
J_{i t} \leq M_{i}\left(1-X_{i t}\right) \quad \forall i \in F, t \in T
$$

$$
\alpha\left(J_{i, t-1}+P_{i t}\right) \leq M_{i}\left(1-X_{i t}\right)+Q_{i} t \quad \forall i \in F, t \in T
$$$$
\sum_{t^{\prime}=t}^{t+g_{\min }} X_{i t^{\prime}} \leq Y_{i} \quad \forall i \in F, t \leq|T|-g_{\min }
$$

$$
\begin{aligned}
\sum_{t^{\prime}=t}^{t+g_{\max }} X_{i t^{\prime}} \geq Y_{i} & \forall i \in F, t \leq|T|-g_{\max } \\
Q_{i t} \leq M_{i} X_{i t} \quad & \forall i \in F, t \in T \\
J_{i 0}=0 & \forall i \in F \\
Y_{i t}, X_{i t} \in\{0,1\} & \forall i \in F, t \in T \\
I_{t}, I_{t}^{\prime}, J_{t}, W_{i t} \geq 0 & \forall i \in F, t \in T
\end{aligned}
$$


The objective function (1) minimizes the total cost which is composed of transportation costs, additional costs of buying biomass from non-contractual suppliers, cost of holding biomass surplus to the requirements of $K$ days and standard inventory costs. Constraints (2) are the inventory balance equations for the plant. Constraints (3) calculate the inventory surplus to the requirement of K days. Constraints (4) balance the inventory at each farm. Constraints (5) permit discarding only in case of a collection. $M_{i}$ is a large number th at can be set at $\sum_{t^{\prime}=0}^{t} P_{i t^{\prime}}$. Constraints (6) indicate that whatever is left after any pickup will be lost and so it will not be considered in the successive periods. Constraints (7) force a minimum amount of biomass to be collected, while constraints (8) and (9) impose the time-windows on the collections. Constraints (10) allow collection only when collection is permitted and only from the selected farms. Another point that must be noted is that the model contains both binary as well as continuous variables. However, the objective function depends only on the continuous variables.

\section{EXACT APPROACH}

\section{A. Case Study}

There is no existing benchmark for the problem. Therefore, we apply the MILP model to a case study corresponding to a biomass processing plant located in central France. The plant can be served by a pre-screened set of farms, out of which a set of optimal farms have to be determined. In this instance, 29 farms were considered for selection. The location of the farms is showed in Figure 1.

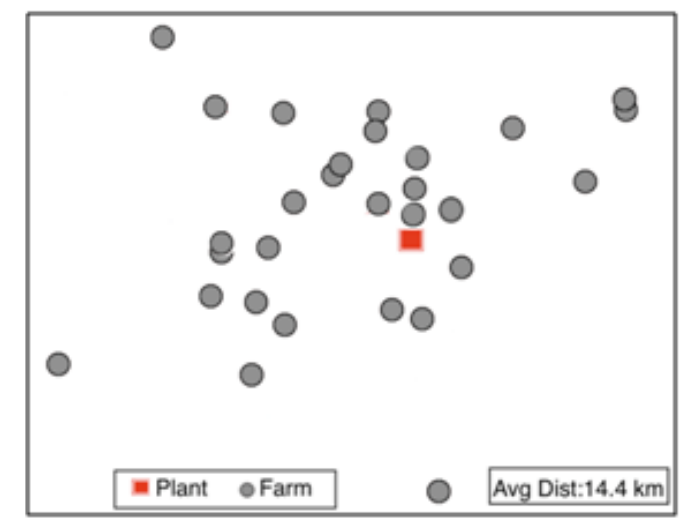

Fig. 1. Location of the 29 pre-screened farms.

For each of these farms, the production of biomass has been forecast based on historical records, which takes into account the inherent seasonality.

The capacity of the plant is considered fixed. However, in order to understand the structure of the problem, several capacity scenarios were studied. In addition to that, the problem was solved with different time discretizations. The various parameters for the problem are detailed in Table III.

\section{B. Computational Results}

Several scenarios were run on CPLEX Optimization Studio 12.6 on a Core i5-3230M CPU 2.60GHz, 6GB RAM. The
TABLE III. PARAMETERS

\begin{tabular}{|c|c|}
\hline Parameter & Value \\
\hline$|F|$ & 29 \\
$g_{\min }$ & 4 weeks \\
$g_{\max }$ & 13 weeks \\
$K$ & 2 weeks \\
$\alpha$ & $85 \%$ \\
$n$ & 7 or 15 \\
$C$ & $60,70,80$ \\
\hline
\end{tabular}

TABLE IV. UPPER BOUND (UB), LOWER BOUND (LB) AND GAP AFTER 3 HOURS FOR SEVERAL VALUES OF C AND N.

\begin{tabular}{cccc}
\hline C & LB & UB & \% Gap \\
\hline \multicolumn{4}{c}{ n=15 (time period of two weeks) } \\
\hline 80 & 51322.25 & 51568.60 & $0.48 \%$ \\
70 & 33670.21 & 34010.28 & $1.01 \%$ \\
60 & 19423.02 & 19720.19 & $1.53 \%$ \\
\hline \multicolumn{4}{c}{ n=7 (time period of 1 week) } \\
\hline 80 & 51958.30 & 53285.70 & $2.49 \%$ \\
70 & 30840.80 & 35187.60 & $12.35 \%$ \\
60 & 16964.1 & 20838.7 & $18.59 \%$ \\
\hline
\end{tabular}

runs were made for a runtime of 3 hours for several values of capacity $\mathrm{C}$ and period lengths $\mathrm{n}$. We analyzed 3 different capacity values, namely, 60 tons/day, 70 tons/day and 80 tons/day. In this particular case, it must be noted that the total availability of manure corresponds to around 70 tons per day. Thus, the three chosen capacities represent the cases of under-consumption, total-consumption and over-consumption, respectively. The results are presented in Table IV.

From Table IV, it can be noted that the practical difficulty of the problem depends on both the time discretization as well as the capacity of the facility. It can be easily understood that the dependence on time discretization is due to the explosion of the number of variables for the same length of the planning horizon. However, the dependence on the capacity is not that straightforward. The instance where the capacity corresponds to the total available manure is easier to solve as it is in the interest of the problem to exclude all solutions which do not include selection of all farms. While the exact method works well for lower temporal resolutions and capacities greater than the availability of the manure, it becomes highly intractable for the other cases.

\section{Adaptive Large Neighborhood Search (ALNS) METAHEURISTIC}

Since the problem becomes intractable for larger instances with finer time discretization, we explore the possibility of solving the problem through a metaheuristic. The Adaptive Large Neighbourhood Search (ALNS) was proposed by Ropke and Pisinger [8] and is a variant of Large Neighbourhood Search (LNS) framework which was first proposed by Shaw [36] to solve Vehicle Routing Problems. The metaheuristic is very similar to the ruin and create heuristic proposed by Schrimpf et al. [37]. An extensive review of the LNS and ALNS methodologies and their applications has been provided in ref. [38]. The basic mechanism of the LNS is to destroy and repair a solution iteratively using a combination of several destroy and repair heuristics. In ALNS, the destroy and repair heuristics are chosen dynamically based on their performance in the solution process. A general scheme of the ALNS framework is depicted in Algorithm 1. 


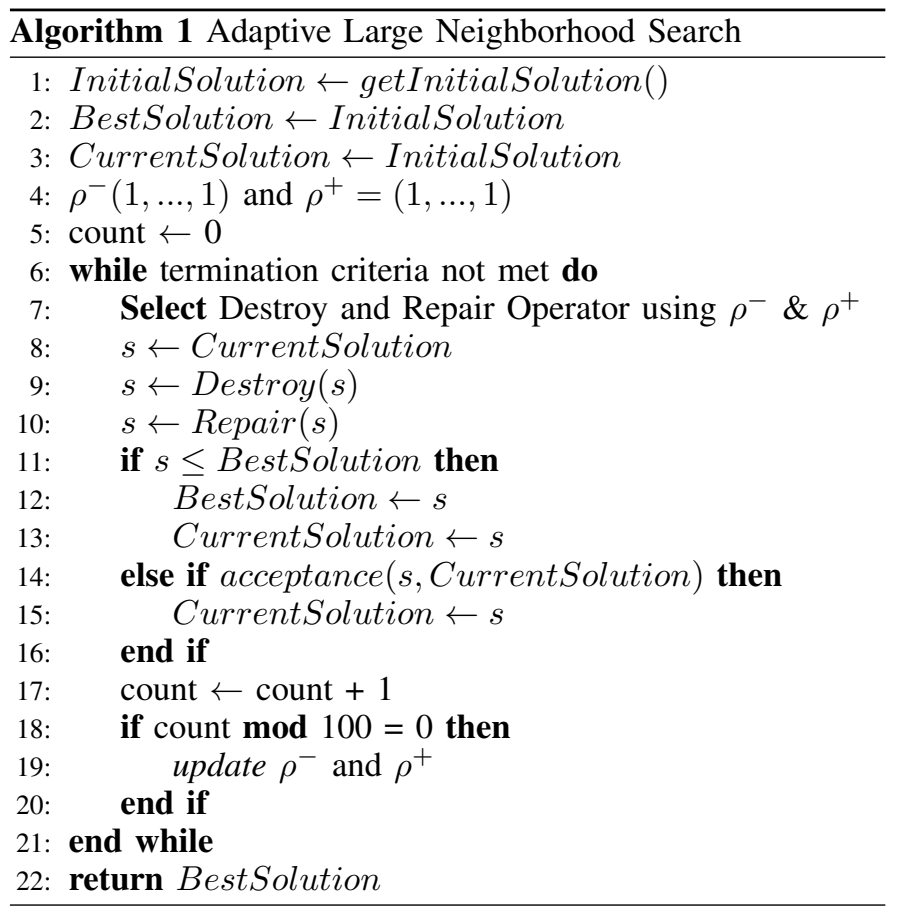

At each iteration, a part of the current solution is removed through some predefined Destroy mechanism to get a partial solution $s$ (line 8). This solution $s$ is then reconstructed using one of the predefined Repair heuristics to reach a new feasible solution (line 9). The solution $s$ can be then accepted or rejected based on acceptance criteria (line 13), which may be defined by the user. The Destroy and Repair mechanisms are selected based on their past performance using scores $\rho^{-}$and $\rho^{+}$, respectively (line 6), which are updated at the end of each 100 iterations (line 16).

\section{A. ALNS Implementation}

The ALNS metaheuristic was coded in Java. It is modular and can be applied to the problem at hand by defining the following problem specific components: initial solution, acceptance criterion, stopping criterion. The various destruction and repair operators will be detailed in the next section.

1) Solution Encoding: A solution can be encoded as a set of three interdependent matrices $Y, X$ and $Q . Y$ is a binary matrix of dimension $|F| \times 1$ representing the selection of farms. $X$ is a binary matrix of dimension $|F| \times|T|$ representing the collection periods at each farm. $Q$ is a real positive matrix of dimension $|F| \times|T|$ representing quantities collected at each period at each farm.

2) Getting Initial Solution: In the initial solution, we consider that all farms are selected. In this situation, the problem can be considered equivalent to a multi-dimensional knapsack problem, where the capacity of the facility has to be filled with supplies from the selected farms. However, in this case, the supplies surplus to the capacity can be stored in the inventory, with inventory cost $H$ and penalties for supplies of more than $K$ days of requirement. We use the greedy mechanism proposed by Dantzig [39] to solve the knapsack problem for each period sequentially. The farms which high quantity of waste per unit distance are preferred. If a farm is selected for one given period, it is not selected during the following $g_{\min }-1$ periods to maintain feasibility. Further, in case a farm is not selected for $g_{m} a x$ periods, then it is selected in the next period. This ensures time constraints satisfaction.

3) Acceptance Criterion: To avoid the solution from getting trapped in a local-minimum, a classical approach is to adopt an acceptance criterion based on the principles of simulated annealing [40]. If solution $s^{\prime}$ deteriorates previous solution $s$, it can be accepted, with probability $e^{-\frac{f\left(s^{\prime}\right)-f(s)}{\theta}}$, where $s$ is the current solution and $\theta$ is a positive parameter called temperature. The initial temperature is set such that a solution that is $5 \%$ worse than the initial solution has an acceptance probability of $50 \%$. This temperature is then reduced at the end of each iteration with a fixed cooling rate of $0.03 \%$.

4) Stopping Criterion: The algorithm is run for 10000 iterations.

\section{B. ALNS Operators}

This section details the operators used in our implementation of the ALNS metaheuristic.

1) Destruction Operators: The destruction operators are used to remove some parts of the solution, so that newer solutions can be explored by re-constructing partial solutions. In this particular problem, we distinguish two ways of destroying a solution:

a) altering the matrix $Y$ of farm selection,

b) altering the matrix $X$ of farm collections.

Let us first describe the destruction operators of family a). Note that after altering matrix $Y$, matrices $X$ and $Q$ must be completely rebuilt.

a1) Random De-Selection: This operator randomly deselects between 1 and $20 \%$ selected farms from matrix $Y_{i}$.

a2) Random Selection: In addition to preserving the selected farms from the incumbent solution, this operator randomly selects between 1 and $20 \%$ of the previously nonselected farms. This allows the construction of new $X$ and $Q$ matrices over a wider range of farms.

a3) Random Toggle This operator randomly chooses between 1 and $0.2 \times|F|$ elements in matrix $Y$ and toggles the values between 0 and 1 . The selected farms are set to 0 , while non selected farms are set to 1 .

a4) Worst Output/Distance Ratio Swap: This operator sorts the lists of selected $(S)$ and non-selected (NS) farms in the ascending order of total output through the planning horizon per distance $\left(\sum_{t} P_{i t} / d_{t}\right)$. Then, the $r^{t h}$ element in the selected list is set as non-selected while the $p_{t h}$ element in the nonselected list is set as selected. The value of $r$ and $p$ are calculated as shown in (14) and (15):

$$
\begin{gathered}
r=S\left[\lambda^{p}|S|\right] \\
p=N S\left[\theta^{p}|N S|\right]
\end{gathered}
$$

where $\lambda$ and $\theta$ are random numbers between 0 and 1 , $p \geq 1$ is a deterministic parameter as described by Ropke and Pisinger [8]. 
a5) Worst Surplus Removal: The last farm added during each period produces a surplus $I_{t}^{\prime}$, which is undesirable. Thus a list $L$ containing the last added farms during each period is maintained, and sorted non-increasingly based on their value of surplus. The $q_{t h}$ element from the list de-selected is presented in (16).

$$
\left.q=L\left[\kappa^{p}|L|\right]\right]
$$

where, $\kappa$ is a random value between 0 and 1 , and $p \geq 1$ is a deterministic parameter as defined in operator $a 4$.

Let us now detail the destruction operators in family $b$ ). For the following operators, we assume that matrix $Y$ is not destroyed.

b1) Random Erase: For this operator, we randomly erase between $40 \%$ and $60 \%$ of the values in matrix $X$. We choose a high range of destroyed values in order to allow the heuristic to explore different solution regions.

b2) Random Erase and Swap: This operator randomly erases $x_{e} \%$ of the solution matrix. Out of the remaining $\left(100-x_{e}\right) \%$ of the matrix, we toggle $15 \%$ of the values. Only feasible toggles are performed, which do not violate the $g_{\min }$ rule. The $g_{\max }$ rule can be compromised in this phase of the algorithm and would be taken care of in the repair phase. While rule $b 1$ allows preserving a part of the solution and building around it, there are still chances of patterns being repeated. The rule $b 2$ forces the solution to have some characteristics opposite to the incumbent solution.

b3) Removal of collections with surplus: This operator forces the value of collections generating surplus to 0 . The removed collections, along with the collections from the nondestroyed solutions, are kept intact for the repair phase.

2) Repair Operators: While the destruction operators allow the algorithm to navigate through a wider section of the solution space, the repair operators follow the path of intensification. They are more focused on reaching the best solution for a given partial solution. The following repair operators were employed during the study:

c1) Greedy Repair This operator is almost the same as the construction heuristic used for the initial solution. However, in case of solutions for which the collections were partially destroyed, we consider the capacity of the period as a partiallyfilled knapsack. Then, we try to fill this capacity by the best feasible collection. The best collection is the collection with the highest value of biomass available per unit distance.

c2) Noised Greedy Repair A noise of $\eta_{i}$, very close to 1, is added to the value $\nu_{i}$ of biomass per unit distance for each farm $i \in F$, such that the updated value for farm each farm is $\eta_{i} \times \nu_{i}$. Now, the knapsack is again filled with the feasible farms with the best noised values.

3) Selecting Destroy and Repair Operators: The destroy and repair operators are selected through an adaptive roulette wheel procedure. Each destroy (or repair) operator $j$ is given a score $\rho_{j}^{-}$(or $\rho_{j}^{+}$) based on its performance during the previous iterations. Higher value of $\rho_{j}^{-}$(or $\rho_{j}^{+}$) suggests greater success of the operator for the given instance. The probability of selecting an operator from $m$ different operators is given as $\frac{\rho_{j}}{\sum_{j=1}^{m} \rho_{j}}$.
TABLE V. COMPARISON OF UPPER BOUNDS ACHIEVED BY CPLEX AND THE ALNS ALGORITHM.

\begin{tabular}{ccccc}
\hline C & CPLEX & LNS & \% Gap & CPU LNS (sec) \\
\hline \multicolumn{5}{c}{$\mathrm{n}=15$ (time period of two weeks) } \\
\hline 80 & 51568.60 & 51523.24 & $0.09 \%$ & 141.4 \\
70 & 34010.28 & 33896.40 & $0.33 \%$ & 139.8 \\
60 & 19720.19 & 19444.14 & $1.40 \%$ & 140.2 \\
\hline \multicolumn{5}{c}{$\mathrm{n}=7$ (time period of 1 week) } \\
\hline 80 & 53285.70 & 52489.30 & $1.49 \%$ & 142.6 \\
70 & 35187.60 & 30897.36 & $12.19 \%$ & 143.6 \\
60 & 20838.70 & 17411.8 & $16.44 \%$ & 141.7 \\
\hline
\end{tabular}

The value of $\rho_{j}$ is updated at the end of each segment. We define a segment as 100 iterations. During each segment, we sum up the scores received by each operator for its contribution to a solution. An operator receives a score $\sigma_{1}$ if it provides a new global best solution, $\sigma_{2}$ if it provides a solution which has never been accepted before with objective value better than the current solution, and $\sigma_{3}$ if it provides a solution which has never been accepted before but with objective value worse than the current solution. Thus, at the end of each segment, the value of $\rho_{j}$ is updated as in equation (17).

$$
\rho_{j} \leftarrow \rho_{j}(1-\eta)+\eta \frac{\pi_{j}}{\Omega_{j}}
$$

where, $\pi_{j}$ is the sum of scores received by operator $j$ during the last segment, and $\Omega_{j}$ is the number of times it was selected. The reaction factor $\eta \in[0,1]$ controls the rate of adjustment of $\rho$.

\section{Assessment of the ALNS metaheuristic}

Due to the stochastic nature of the heuristic, 10 runs for each scenario were made and the average computational results are provided in Table $\mathrm{V}$. Columns 2 and 3 report the upper bounds obtained with Cplex (after 3 hours of computation) and the ALNS metaheuristic, respectively. Column 4 is the relative difference between the results on Columns 2 and 3. Column 5 indicates the computation time for the 10000 iterations of the ALNS.

It can be seen that the ALNS method shows significant improvement over the solution provided by CPLEX as we move towards the more constrained instances. The improvement is as high as $16 \%$ with capacity 60 and time discretization of 7 days. Thus, it can be seen that ALNS, in general, provides better and quicker solutions.

\section{CONClusion AND Future WORK}

The optimization of supply chain for biopower production from animal waste is a relatively unexplored field from an optimization research point of view. In this study we address the optimization of biopower supply chains with animal waste as the source of energy. We propose a mathematical model which takes into account the practical constraint of collection time-windows imposed by the inherent nature of the animal waste. We also consider other practical constraints which often tie facilities into collecting a minimum proportion of the total production. Then, we propose an ALNS metaheuristic that can solve the problem very efficiently.

The proposed model can be further improved by inclusion of transportation fleet management and a finer modeling of 
methane loss. Possible extensions include mixed sources of biomass (animal, energy crops, wood, industrial waste, etc.) or bi-objective optimization of both the logistic costs and the environmental impact.

\section{REFERENCES}

[1] European Commission, "Energy - Country factsheets version 3.0," ec.europa.eu/energy/sites/ener/files/documents/2014-countryfactsheets.pdf, 2014, (Accessed july 22nd 2015).

[2] Netherlands Enterprise Agency, "Market opportunities for biomass in France," english.rvo.nl/sites/default/files/2014/06/Country Monitor France - Final_April 2014.pdf, April 2014, (Accessed july 22nd 2015).

[3] J. B. H. Nielsen and P. Oleskowicz-Popiel, "The future of biogas in Europe: Visions and targets until 2020," in Proceedings of the European Biogas Workshop, Esbjerg, Denmark, 2007.

[4] ecoprog, Envalys, "Biogas plants in France market and new site potentials," www.ecoprog.com/en/publications/energy-industry/biogasplants-france.htm, 2014, (Accessed july 22nd 2015).

[5] A. Kavkler, S. Repina, and M. Festić, A Comparison of Electricity Generation Reference Costs for Different Technologies of Renewable Energy. InTechOpenpen, 2012, ch. 14, pp. 309-318.

[6] J. N. de Figueiredo and S. F. Mayerle, "A systemic approach for dimensioning and designing anaerobic bio-digestion/energy generation biomass supply networks," Renewable Energy, vol. 71, pp. 690-694, 2014.

[7] D. Pisinger and S. Ropke, "A general heuristic for vehicle routing problems," Computers \& Operations Research, vol. 34, no. 8, pp. 24032435, 2007.

[8] S. Ropke and D. Pisinger, "An adaptive large neighborhood search heuristic for the pickup and delivery problem with time windows," Transportation Science, vol. 40, no. 4, pp. 455-472, 2006.

[9] B. Sharma, R. Ingalls, C. Jones, and A. Khanchi, "Biomass supply chain design and analysis: basis, overview, modeling, challenges, and future," Renewable \& Sustainable Energy Reviews, vol. 24, pp. 608-627, 2013.

[10] F. Mafakheri and F. Nasiri, "Modeling of biomass-to-energy supply chain operations: applications, challenges and research directions," Energy Policy, vol. 67, pp. 116-126, 2014.

[11] S. Gold and S. Seuring, "Supply chain and logistics issues of bio-energy production," Journal of Cleaner Production, vol. 19, pp. 32-42, 2011.

[12] M. Eskandarpour, P. Dejax, J. Miemczyk, and O. Péton, "Sustainable supply chain network design: an optimization-oriented review," Omega, vol. 54, pp. 11-32, 2015.

[13] B. Sharma, R. G. Ingalls, C. L. Jones, R. L. Huhnke, and A. Khanchi, "Scenario optimization modeling approach for design and management of biomass-to-biorefinery supply chain system," Bioresource Technology, pp. 163-171, 2013.

[14] D. Yue, F. You, and S. W. Snyder, "Biomass-to-bioenergy and biofuel supply chain optimization: Overview, key issues and challenges," Computers \& Chemical Engineering, pp. 36-56, 2014.

[15] S. D. Eksioglu, A. Acharya, L. E. Leightley, and S. Arora, "Analyzing the design and management of biomass-to-biorefinery supply chain," Computers \& Industrial Engineering, pp. 1342-1352, 2009.

[16] T. Lin, L. F. Rodríguez, Y. N. Shastri, A. C. Hansen, and K. C. Ting, "Integrated strategic and tactical biomass-biofuel supply chain optimization," Bioresource Technology, vol. 156, pp. 256-266, 2014.

[17] J. Zhang, A. Osmani, I. Awudu, and V. Gonela, "An integrated optimization model for switchgrass-based bioethanol supply chain," Applied Energy, vol. 102, pp. 1205-1217, 2013.

[18] I. Tatsiopoulos and A. Tolis, "Economic aspects of the cotton-stalk biomass logistics and comparison of supply chain methods," Biomass \& Bioenergy, vol. 24, no. 3, pp. 199-214, 2003.

[19] Y. Shastri, A. Hansen, L. Rodríguez, and K. Ting, "Optimization of miscanthus harvesting and handling as an energy crop: Biofeed model application," Biological Engineering, vol. 3, no. 2, pp. 37-69, 2010.

[20] A. A. Rentizelas, A. J. Tolis, and I. P. Tatsiopoulos, "Logistics issues of biomass: The storage problem and the multi-biomass supply chain," Renewable \& Sustainable Energy Reviews, vol. 13, pp. 887-894, 2009.
[21] J. Kim, M. J. Realff, J. H. Lee, C. Whittaker, and L. Furtner, "Design of biomass processing network for biofuel production using an MILP model," Biomass and Bioenergy, vol. 35, no. 2, pp. 853-871, 2011.

[22] Ş. Y. Balaman and H. Selim, "A network design model for biomass to energy supply chains with anaerobic digestion systems," Applied Energy, vol. 130, 2014.

[23] M. Svanberg, I. Olofsson, J. Flodén, and A. Nordin, "Analysing biomass torrefaction supply chain costs," Biosource Technology, vol. 142, pp. 287-296, 2013.

[24] J. N. de Figueiredo et S. F. Mayerle, "A systemic approach for dimensioning and designing anaerobic bio-digestion/energy generation biomass supply networks," Renewable Energy, vol. 71, pp. 690-694, 2014.

[25] G. Laporte, R. Musmanno, and F. Vocaturo, "An adaptive large neighbourhood search heuristic for the capacitated arc-routing problem with stochastic demands," Transportation Science, vol. 44, no. 1, pp. 125$135,2010$.

[26] R. Masson, F. Lehuédé, and O. Péton, "An adaptive large neighborhood search for the pickup and delivery problem with transfers," Transportation Science, vol. 47, no. 3, pp. 344-355, 2013.

[27] S. Kristiansen and T. R. Stidsen, "Adaptive large neighborhood search for student sectioning at Danish high schools," in Proceedings of the $9^{\text {th }}$ International Conference on the Practice and Theory of Automated Timetabling (PATAT 2012), 2012.

[28] N. Azia, M. Gendreau, and J. y. Potvin, "An adaptive large neighborhood search for a vehicle routing problem with multiple routes," Computers \& Operations Research, vol. 41, pp. 167-173, 2014.

[29] E. Demir, T. Bektas, and G. Laporte, "An adaptive large neighborhood search heuristic for the pollution-routing problem," European Journal of Operational Research, vol. 223, no. 2, pp. 346-359, 2012.

[30] M. Sorensen, S. Kristiansen, and T. R. Stidsen, "International timetabling competition 2011: An adaptive large neighborhood search algorithm," in 9th International Conference on the Practice and Theory of Automated Timetabling (PATAT 2012), 2012.

[31] J. Korsvik, K. Fagerholt, and G. Laporte, "A large neighbourhood search heuristic for ship routing and scheduling with split loads," Computers \& Operations Research, vol. 38, no. 2, pp. 474-483, 2011.

[32] M. Eskandarpour, P. Dejax, and O. Péton, “A large neighborhood search heuristic for supply chain network," Ecole des Mines de Nantes, Technical report AUTO/14.3, 2014.

[33] P. Copado-Méndez, C. Blum, G. Guillén-Gosálbez, and L. Jiménez, "Large neighbourhood search applied to the efficient solution of spatially explicit strategic supply chain management problems," Computers and Chemical Engineering, vol. 49, pp. 114-126, 2013.

[34] B. Batidzirai, F. van der Hilst, H. Meerman, M. Junginger, and A. P. C. Faaij, "Optimisation potential of biomass supply chains with torrefaction technology," Biofuels, Bioproducts and Biorefining, vol. 8, no. 2, pp. 253-282, 2014.

[35] V. Dornburg, D. van Vuuren, G. van de Ven, and H. L. et al., "Bioenergy revisited: key factors in global potentials of bioenergy," Energy \& Environmental Science, vol. 49, pp. 258-267, 2010.

[36] P. Shaw, "Using constraint programming and local search methods to solve vehicle routing problems," Lecture Notes in Computer Science, vol. 1520 , pp. 417-431, 1998.

[37] G. Schrimpf, J. Schneider, H. Stamm-Wilbrandt, and G. Dueck, "Record breaking optimization results using the ruin and recreate principle," Journal of Computational Physics, vol. 159, pp. 139-171, 2000.

[38] D. Pisinger and S. Ropke, "Large neighbourhod search," in Handbook of Metaheuristics, U. Springer, Ed., 2010, pp. 399-419.

[39] G. B. Dantzig, "Discrete-variable extremum problems," Operations Research, vol. 5, no. 12, pp. 266-288, 1957.

[40] S. Kirkpatrick, C. D. Gelatt, and M. Vecchi, "Optimization by simulated annealing," Science, New Series, vol. 220, no. 4598, pp. 671-680, 1983. 